\title{
Complete Globe Protrusion Post Trauma: A Case Report
}

\author{
R.C.Gupta ${ }^{1}$, Priyanka Gupta ${ }^{1}$ \\ ${ }^{I}$ (Department of ophthalmology, GSVM Medical college, India)
}

\begin{abstract}
Complete traumatic protrusion of the globe is a rare event, and there are only a few reported cases in the literature. Complete globe protrusion post trauma may be associated with optic nerve avulsion where visual recovery is obviously not expected. We report a case of 9 year old boy who was a victim of bicycle accident with unilateral globe protrusion. Vision in the protrudedeye was reduced to perception of light with gross restriction of ocular movements. After repositioning the globe andtarsorrhaphy patient showed satisfactory visual and cosmetic improvement. Thus good visual recovery and ocular motility may be achieved by prompt and appropriate management in traumatic protruded globe.
\end{abstract}

Keywords- complete globe protrusion, trauma

\section{Introduction}

Completeprotrusion of the globe with or without optic nerve avulsion is a rare condition that results from an extreme form of facial or orbital trauma.[1] The optic nerve and the globes are very resistant to mild and moderate trauma.[2] The existing literature indicates that either optic nerve avulsion or extraocular muscles avulsion separately or both occur. ${ }^{2}$ In our case, inspite of full protrusion of globe neither of these was involved resulting in satisfactory salvage of globe integrity and visual outcome.

\section{Case Report}

We have conducted our study keeping in mind the Helsinki Declaration of 1975, as revised in 2000. A nine year old boy presented to the emergency department withhistory of injury by bicycle handle approximately 6 hours ago.

Onexamination left globe was found displaced anteriorly and posterior part of globe was caught tightly by the closed lids (Fig. 1). Conjunctiva was hyperemic with few bluish discolored patches suggesting anterior segment ischemia. Cornea showedearly signs of exposure keratopathy.Pupil was dilated andsluggishly reacting. The movements of left eye were grossly restricted in allgazes. Visual acuitywas reduced to lightperception. Right eye was normal (Fig. 1).

The patient was hospitalized and put on intravenous corticosteroids (1 milligram methylprednisolone per kilograms of body weight)to take care of possible nerve compression and prophylactic antibiotics.

An urgent MRI scan wasperformed which showed protrusion of the left globe with intact orbital walls. Optic nerve did not show any avulsion. Intracranial structures were normal. The right eye was normal.

Patient was urgently taken for surgery the next day under general anesthesia. An attempt to reposit the protruded eyeball was unsuccessful. Therefore lateral canthotomy was done. Finger was inserted from canthotomy site into the orbit and a tight girdle of tenon was felt behind the globe on palpation. After orbital exploration multiplerelaxing incisions in girdle of tenon were given to reposit the globe. After repositioning the globe, muscles and their insertions were carefully examined and found intact. The lacerated conjunctiva was repaired and central tarsorrhaphy was done which was released after 15 days. After three months post operatively globe was inposition (Fig. 2). The ocular movements and best corrected visual acuity returned to nearnormal (visual acuity 6/9). Color vision and contrast sensitivity was also normal.

\section{Discussion}

Complete globe protrusion is a rare condition usually resulting from severe trauma to the orbit and face. It may be associated with either optic nerve is injury or disruption of theextraocularmuscles along with optic nerve. Obviously if optic nerve is avulsed, visual recovery may not be expected. Following trauma, the most commonly injured and avulsedextraocular muscles in decreasing order of frequency are the medialrectus, inferior rectus, superior rectus, and the obliques.[3] It may lead to life-threatening neurologic sequel like orbital infection, intracranial or subarachnoid hemorrhage, cerebrospinalfluid leakage, meningitis, or hypothalamic infarcts. There are many school of thought regarding management ofprotruded globe. It has beensuggested that reoperation on a lost muscle should be performed within 7 to 10 days after the initial surgery since contracture ofmuscles may occur.[4] Some of the reportedcases of avulsed globe were replaced within theorbit without later requirement of enucleation. $[1,5]$ On the other handsome were initially repositioned but subsequently needed enucleation.[6]No report of sympathetic ophthalmia was reported in these cases. Prompt reposition of the globe 
in comparison with initial enucleation, has two advantages; first, the patient has not to sacrifice an organ after such severe accident; secondly, we can easily fit an ocular prosthesis with better motility on phthisic eye.

In our case neither optic nerve nor extraocular muscles were avulsed, therefore ocular movements and visual acuity along with globe could be saved satisfactorily (Fig. 2). This probably happened because of early and correct intervention and fortunate globe protrusion merely due to stretching of extraocular muscles and retroorbital tissue. Immediate intravenous corticosteroids were started to take care of possible nerve compression as the pupil on presentation was dilated and sluggishly reacting. Central tarsorrhaphy probably helped to maintain globe and other tissues in their respective anatomical positions.

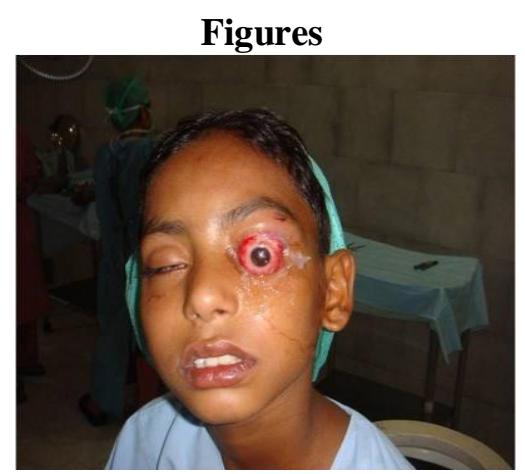

Fig.1:Preopertive

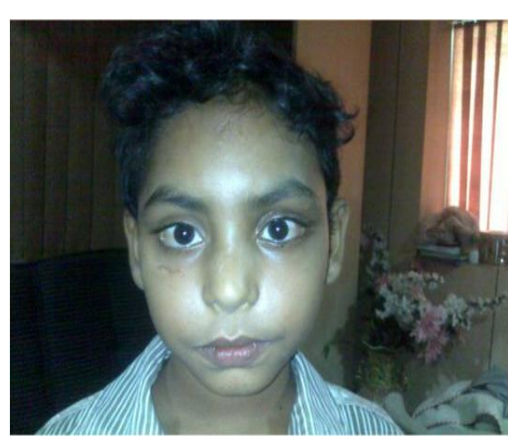

Fig. 2: Three months post-operative

\section{Conclusion}

In globe protrusion, proper assessment of severity and extent of tissue involvement is very crucial. Canthotomy/ cantholysis, releasing the girdle of tenon, globe reposition at its place and complete tarsorrhaphy helps in keeping the globe in position. A prompt decision and sequential management may result in good visual and cosmetic outcome in traumatic globe protrusion if vital ocular structures are not severely compromised.

\section{Acknowledgements}

R.N.Kushwaha, Assistant Professor,Department Of Ophthalmology, G.S.V.M. Medical College, India.

\section{References}

[1]. Bajaj MS, Kedar S, Sethi A. Traumatic globe luxation with optic nerve transection. Orbit. 2000;19:165-70.

[2]. Unal S, Argin A, Arslan E, et al. Bilateral complete avulsion of ocular globes in a Le Fort III maxillofacial fracture: a case report and review of the literature. Eur J Ophthalmol. 2005;15:123-5.

[3]. Mailer CM. Avulsion of the inferior rectus. Can J Ophthalmol. 1974;9:262-6.

[4]. Kiratli H, Tümer B, Bilgic S. Management of traumatic luxation of the globe. A case report. Acta Ophthalmol Scand. 1999; 77:3402 .

[5]. Song A, Carter KD. Bilateral traumatic globe subluxation. Ophthal Plast Reconstr Surg. 2006;22:136-7.

[6]. Lelli GJ, Jr, Demirci H, Frueh BR. Avulsion of the optic nerve with luxation of the eye after motor vehicle accident. Ophthal Plast Reconstr Surg. 2007;23:158-60. 